\title{
Interdisciplinariedad entre educación física y ciencias naturales para mejorar aprendizajes en niñas de tercer grado de educación básica
}

\author{
Jose Salvador Pagano Bigio¹, Carlos Alberto Pérez Guardo² \\ Recibido: 02-05-2015 - Aceptado: 15-06-2015
}

\begin{abstract}
Resumen
Determinar la efectividad de la interdisciplinariedad entre la Educación Física y las Ciencias Naturales en el mejoramiento de los aprendizajes en niñas de tercer grado de básica primaria es el elemento esencial sobre el cual se desarrolla la presente investigación, siendo el término "interdisciplinariedad" aquel que se aplica en el campo pedagógico al trabajo científico que requiere metodológicamente de, por lo menos, dos disciplinas; brinda nuevas estrategias didácticas al servicio de los docentes y del proceso enseñanza-aprendizaje, centrándose en la interacción entre asignaturas. La Educación Física y las Ciencias Naturales mantienen una relación en ciertos contenidos de enseñanza, facilitando la interdisciplinariedad entre ellas. En el presente estudio se trabajó con un grupo control con el cual se desarrollaron clases interdisciplinares y un grupo contraste, que aprendió bajo el esquema metodológico de la institución; siendo una investigación de paradigmas cuantitativo y cualitativo y que se desarrolla bajo el método cuasi-experimental y evaluada desde lo conceptual, psicomotor y socio-afectivo. Se concluye que el modelo de conexión planteado por Fogarty, aplicado entre la Educación Física y las Ciencias Naturales, permite el aprendizaje significativo de estas disciplinas, mejorando el promedio de notas en 1.1 puntos mediante el desarrollo de conocimientos integrados a través de unidades interdisciplinares.
\end{abstract}

Palabras clave: interdisciplinariedad, educación física, ciencias naturales, aprendizaje.

\section{Interdisciplinarity between physical education and natural sciences to improve learning in third grade girls basic education}

\begin{abstract}
To determine the effectiveness of interdisciplinarity between Physical Education and Natural Sciences on improving learning in third grade girls basic education is the essential element on which this research is conducted, being interdisciplinarity term the one that is applied in education field to the scientific work that requires methodologically at least two disciplines; it provides new teaching strategies at the service of teachers and teaching-learning process focusing in the interaction between subjects. Physical Education

\footnotetext{
${ }^{1}$ Magíster en educación, especialista en pedagogía del deporte para menores, licenciado en Educación Física. Docente en Corporación Universidad de la Costa, Barranquilla, Colombia; pepepag@hotmail.com

${ }^{2}$ Magíster en educación, especialista en pedagogía del deporte para menores, licenciado en Educación Física. Docente en
} Corporación Universidad de la Costa, Barranquilla, Colombia; carpg02@hotmail.com
\end{abstract}


BÚSQUEDA - Enero / Junio de 2015 - No. 14 (77 - 83)

and natural science maintain a relationship in certain teaching contents facilitating interdisciplinarity between them, for this purpose we worked with a control group which were developed interdisciplinary classes and a contrast group who worked under the normal scheme of the institution; being a qualitative - quantitative research in which it develops under the quasi-experimental method, evaluated from the conceptual, psychomotor, social and emotional. Allowing conclude the connection model posed by Fogarty, applied between physical education and natural sciences allows meaningful learning of these disciplines improving grade point average in 1.1 points by developing integrated knowledge through interdisciplinary units.

Keywords: interdisciplinary, physical education, natural sciences, learning.

\section{Introducción}

La interdisciplinariedad es una herramienta efectiva para lograr un mejor aprendizaje; esta brinda nuevas estrategias didácticas al servicio de los docentes y del proceso enseñanzaaprendizaje, generando un cambio en cuanto al modelo tradicional de educación y un mayor y mejor impacto en los estudiantes, motivándolos a actuar con responsabilidad en sus tareas académicas, siendo más competentes. De igual manera la interdisciplinariedad involucra grupos de investigadores, estudiantes y maestros con el objetivo de vincular e integrar muchas escuelas de pensamiento, profesiones o tecnologías.

Fogarty (1991) referencia tres modelos didácticos para el desarrollo de la interdisciplinariedad como son: los modelos de conexión, compartido y de asociación. Para esta investigación solo se utilizó el modelo de conexión, el cual permite relacionar dos o más asignaturas a través de un tema, un concepto o un ejercicio; como se realizó ampliamente durante el desarrollo del trabajo, donde se seleccionaron temas a fines de las Ciencias Naturales y de la Educación Física; todo esto se fundamenta en la búsqueda del desarrollo de las experiencias de aprendizaje.

Otra opinión es la de León (1997) quien sostiene que la interdisciplinariedad tiene un sin número de manifestaciones en la didáctica como son el objeto de estudio, que es realmente el proceso que lleva el maestro para darle solución al problema. Se tiene en cuenta en este proceso principalmente al portador del problema que es el alumno, su realidad y su contexto; se tienen aspiraciones que son precisamente los objetivos a alcanzar, también los conocimientos y las habilidades y valores que el estudiante debe conseguir; siendo el método la forma como se ha de lograr todo lo anterior, teniendo en cuenta sin lugar a duda el medio como soporte indispensable y, por último, la evaluación que es la que dice si el objeto problema ha sido superado.

Frega (2007) manifiesta que la interdisciplinariedad exigirá el logro de relaciones de reciprocidad o co-implicación entre las didácticas de cada disciplina, aceptando que el conocimiento se construye como una estructura y la programación de la enseñanza se basa en una didáctica constructivista que le permite al sujeto cognoscente relacionar sus conocimientos. En esta relación disciplina-enseñanza-aprendizaje la didáctica es el nexo entre ambas estructuras.

La interdisciplinariedad rompe con los límites de cada disciplina, pero le da igual importancia a cada una, permitiendo una complementariedad entre ellas y facilita el proceso de aprendizaje, de manera que se entiende un problema de aprendizaje como una sola cosa. La interdisciplinariedad no es la sumatoria de conocimiento, es más que eso; es la integración de conocimientos, la articulación de disciplinas para producir soluciones a los diferentes problemas de aprendizaje, acaba con las posiciones individualistas de las áreas, permite un currículo más articulado y mejor dispuesto para el conocimiento globalizado; la interdisciplinariedad permite la concertación, el entendimiento, la armonía para el beneficio de la escuela, el alumno y el medio ambiente. 
Jose Salvador Pagano B., Carlos Alberto Pérez G. - Interdisciplinariedad entre educación física y ciencias naturales

Al asumir la práctica de la interdisciplinariedad en la escuela, se quiere promover un enfoque de desarrollo, que no solo busca el crecimiento, sino el desarrollo humano integral, formando continuamente a los ciudadanos no solo en sus habilidades cognitivas o destrezas manuales, sino formando en valores solidarios y cooperativos.

Piaget (1978) argumentó que la interdisciplinariedad es el segundo nivel de asociación entre disciplinas donde la cooperación entre las mismas lleva a interacciones reales; es decir, una verdadera reciprocidad de intercambio $\mathrm{y}$, por consiguiente, enriquecimientos mutuos. Para Piaget, las relaciones interdisciplinarias tienen su base en las propias relaciones epistemológicas que se establecen entre las disciplinas, que consiste en significar sus fundamentos conceptuales y sus leyes para dar soluciones a problemas que se revelan en la escuela.

Conde, Arteaga y Viciano (2011) publicaron en España en la revista Apunts No 51, Educación física y deportes, el artículo titulado "Interdisciplinariedad de las áreas en educación primaria" en el que se afirma que la educación física es refuerzo del área de lengua castellana y literatura. Se desarrollan los objetivos de la educación física, teniendo en cuenta la posibilidad de desarrollar objetivos de otras áreas, planteando la evaluación y los contenidos de la educación física por encima de las demás áreas que se vean reflejadas en las actividades propuestas.

Por otra parte Mertz y Aranda (2010) realizan una investigación en Brasil en la Universidad de Cruz Alta sobre formación docente reflexiva y perspectivas interdisciplinarias en Educación Física. La finalidad del presente estudio se refiere a la forma de cómo se desarrolla la formación de docentes, en el curso de Educación Física, en la Unicruz (Universidad de Cruz Alta, Brasil); el análisis de la práctica docente, así como la evaluación de una propuesta pedagógica interdisciplinar. Con esto se amplía la necesidad de preparar a los docentes para desarrollar las clases de manera interdisciplinar, logrando demostrar la importancia que el maestro se prepare desde esta perspectiva.
De la misma manera, Meriño y Cabezas (2010) destacan en su investigación la importancia de la formación de los docentes de básica primaria en la interdisciplinariedad, como una forma de favorecer su quehacer cotidiano. En esta línea, el investigador Carvajal (2010) plantea la importancia de la interdisciplinariedad en la educación superior y un reto para la investigación en su resumen.

Teniendo en cuenta lo anterior, se plantea el problema de investigación ¿cómo la interdisciplinariedad de la Educación Física y las Ciencias Naturales mejora los aprendizajes en niñas de tercer grado de básica primaria? A partir del modelo de conexión en el cual los contenidos y habilidades y destrezas de la educación física pasan a ser la base para la enseñanza de las unidades y contenidos del área de ciencias naturales, utilizados para mejorar, ampliar y complementar el aprendizaje, lo que lleva a plantear lo siguientes objetivos específicos:

Desarrollar el modelo interdisciplinario de conexión, entre la Educación Física y Ciencias Naturales que permitan el progreso conceptual, psicomotor y socio-afectivo de estas disciplinas en los niños de tercer grado de básica primaria, en la institución educativa Instituto Técnico de Comercio de Barranquilla.

Determinar el rango de superación en el dominio de los conceptos básicos de la Educación Física y Ciencias Naturales que permitan el desarrollo conceptual de estas disciplinas en los niños de tercer grado de básica primaria, en la institución educativa Instituto Técnico de Comercio de Barranquilla.

Proponer de manera sistemática unidades de formación integradas entre Educación Física y Ciencias Naturales para el mejoramiento de los aprendizajes de los niños.

Es importante tener en cuenta la relación que existe entre la Educación Física y las Ciencias Naturales entorno al cuerpo humano, estableciendo una correlación directa, facilitadora, que permita construir una interdisciplinariedad, 
BÚSQUEDA - Enero / Junio de 2015 - No. 14 (77 - 83)

siendo primordial investigarla sobre la base del método científico y de categorías que permitan identificar la incidencia de esta práctica en el aprendizaje.

La Educación Física, como área integradora dentro del currículo, posee unas características particulares que le permiten acercarse a otras áreas del conocimiento; de esta manera, facilita el proceso y la implementación del enfoque interdisciplinar con las demás asignaturas del conocimiento humano; reconociendo la importancia que esta merece en el mejoramiento de los aprendizajes, haciéndolo significativo desde las competencias. Dentro de este contexto investigativo, el tema de la interdisciplinariedad toma mucha importancia, pues, permite ahondar en aspectos propios de este método didáctico y su aplicabilidad en áreas como la educación física y las ciencias naturales, convirtiéndola en una forma de innovación curricular que mejoraría el aprendizaje de las alumnas.

Luego de abordar la temática de la investigación, se estableció el siguiente objetivo general:

Determinar la efectividad de la interdisciplinariedad entre la Educación Física y las Ciencias Naturales en el mejoramiento de los aprendizajes en niñas de tercer grado de básica primaria.

\section{Metodología}

El diseño de investigación hizo referencia al propósito de la maniobra escogida para responder a la pregunta de investigación, lo que se desarrolló para alcanzar el objetivo, en este caso el diseño fue cuasi-experimental, ya que se manipuló una categoría independiente que fue la interdisciplinariedad para ver su consecuencia y correlación con las categorías dependientes que fueron el aprendizaje de la Educación Física y de las Ciencias Naturales de las niñas de 8 y 9 años de tercer año de básica primaria del Instituto Técnico de Comercio de la ciudad de Barranquilla, de las cuales se escogió como muestra el 100\%.
Se conformaron dos grupos; salón 302, grupo de control y salón 301, grupo de contraste integrados por 41 alumnas. Al grupo control se le dictaron cuatro clases interdisciplinares de Educación Física y Ciencias Naturales, las cuales conforman la unidad interdisciplinar. "Descubro mi cuerpo" incluye las partes del cuerpo y el sistema óseo; espacio-tiempo y los movimientos de la tierra; el control respiratorio y el sistema respiratorio; el ajuste postural y el sistema articular. Al grupo contraste se le impartió la clase de Ciencias Naturales de manera tradicional con los mismos contenidos. Se llevó a cabo una investigación cualitativa-cuantitativa. Para la evaluación de los conceptos básicos abordados en clase se aplicó un cuestionario de escogencia múltiple y de asociación. Para las demás competencias se aplicó una ficha de observación a los grupos de control y contraste, utilizando los videos para esto.

Se tuvo en cuenta para la escogencia de los temas desarrollados y para las evaluaciones, los lineamientos curriculares para el grado tercero del Ministerio de Educación Nacional, permitiendo mediante esta guía desarrollar los indicadores de evaluación, en cada una de las competencias tenidas en cuenta, como son la cognitiva, psicomotora y socio-afectiva.

A continuación, se entrará a determinar las categorías de estudios dependientes e independientes escogidas para esta investigación.

Se considera que la interdisciplinariedad entre la Educación Física y las Ciencias Naturales en las niñas de tercer grado de básica primaria es la categoría dependiente en este estudio, ya que es la categoría que se puede manipular y controlar. Se consideró como categoría independiente la caracterización de las niñas de tercer grado de básica primaria de la institución desde la perspectiva de su desarrollo cognitivo, psicomotor y socio-afectivo, el cual es normal para su edad.

A partir de las evaluaciones y de las fichas de observación se procedió a sistematizar la información del grupo control y del grupo 
Jose Salvador Pagano B., Carlos Alberto Pérez G. - Interdisciplinariedad entre educación física y ciencias naturales

contraste, tabulando los resultados de la misma y siguiendo los rangos establecidos por el sistema institucional de evaluación educativo, para posteriormente comparar, establecer las diferencias y determinar los resultados.

\section{Resultados}

Promediando las calificaciones del total de los alumnos en cada competencia y en cada grupo se obtienen los resultados que se manifiestan en la hipótesis, ver tabla No 1:

Tabla 1. Resultados promedio competencias

\begin{tabular}{lccc}
\hline \multicolumn{1}{c}{ COMPETENCIAS } & CONTROL & CONTRASTE & DIFERENCIA \\
\hline CONCEPTUAL & 4,7 & 3,3 & 1,4 \\
SOCIO-AFECTIVO & 4,4 & 2,6 & 1,7 \\
PSICOMOTOR & 4,5 & 4,3 & 0,2 \\
PROMEDIO & 4,5 & 3,4 & 1,1 \\
\hline
\end{tabular}

Fuente: Elaboración propia

Se puede apreciar en el gráfico 1, que en la competencia conceptual, la calificación de todos los alumnos en el grupo de control dio como resultado un promedio de 4,7; mientras el grupo contraste dio un promedio de 3,3 ; indicando una diferencia de 1,4; correspondiendo esta a una diferencia significativa.

Figura 1. Calificaciones promedio competencia conceptual por grupo.

Fuente: Elaboración propia

\section{COMPETENCIA CONCEPTUAL EN TERMINO DE CALIFICACIÓN}

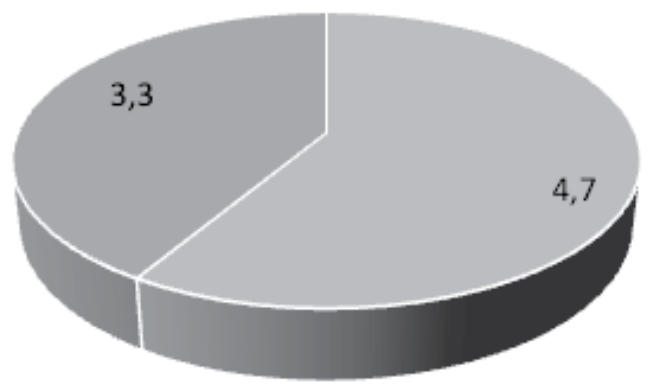

$\|$ CONTROL $=$ CONTRASTE
En el gráfico 2, se observa que el grupo control en las competencias socio-afectiva el promedio general de notas fue de 4,4; en el grupo control competencias socio/efectivas mientras que en el grupo contraste el promedio fue de 2,6, mostrando una diferencia 1,8 entre los dos grupos, enseñando una diferencia significativa.

Figura 2. Calificaciones promedio competencia socioafectiva por grupo

Fuente: Elaboración propia

\section{COMPETENCIA SOCIO-AFECTIVA EN TERMINO DE CALIFICACIÓN}

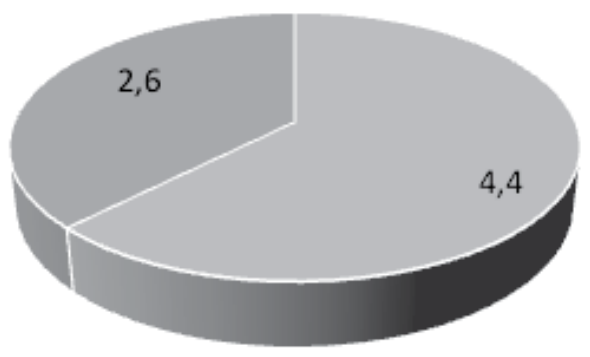

" CONTROL $=$ CONTRASTE 
BÚSQUEDA - Enero / Junio de 2015 - No. 14 (77 - 83)

El grupo control tiene un promedio general de calificación de 4,5 y el grupo contraste, un promedio 4,3. Como muestra el gráfico 3, esto indica una diferencia solo del 0,2 , siendo la única competencia en la que la diferencia no es realmente significativa.

Figura 3. Calificaciones promedio competencia psicomotora por grupo.

Fuente: Elaboración propia

\section{COMPETENCIA PSICOMOTORA EN TERMINO DE CALIFICACIÓN}

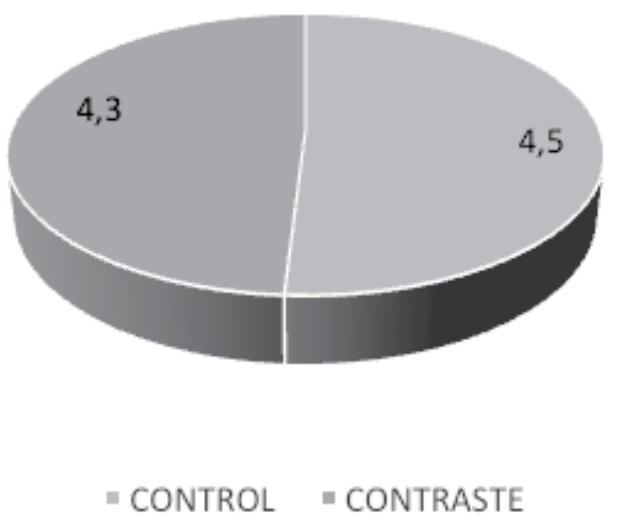

Conclusiones

El modelo interdisciplinario de conexión, aplicado entre la Educación Física y Ciencias Naturales permite el aprendizaje significativo en lo conceptual, psicomotor y socio-afectivo de estas disciplinas en los niñas de tercer grado de básica primaria, acorde a lo investigado en la institución educativa Técnico de Comercio de Barranquilla, mediante la elaboración de un plan clase que permita conjugar de manera no solo interdisciplinar, sino transversalmente todas las competencias sin necesidad de una especificidad en cada una de ellas ni validando el modelo. La clase interdisciplinar permite un mejor ambiente de trabajo, mucho más gratificante para el docente y los alumnos, ya que genera una mayor motivación, dando como resultado un aprendizaje con mayor sentido y significación.
Finalmente, se puede apreciar en el gráfico 4 , de promedio de las tres competencias, que el grupo control tiene un promedio general de $4,5 \mathrm{y}$ el grupo contraste, un promedio 3,4; quedando una diferencia de 1,1 ; siendo esto una diferencia significativa en general 0,1 por encima de la proyectada en la hipótesis.

Figura 4. Calificaciones promedio de las tres competencias.

Fuente: Elaboración propia

\section{PROMEDIO DE LAS TRES COMPETENCIAS EN TERMINOS DE CALIFICACIÓN}

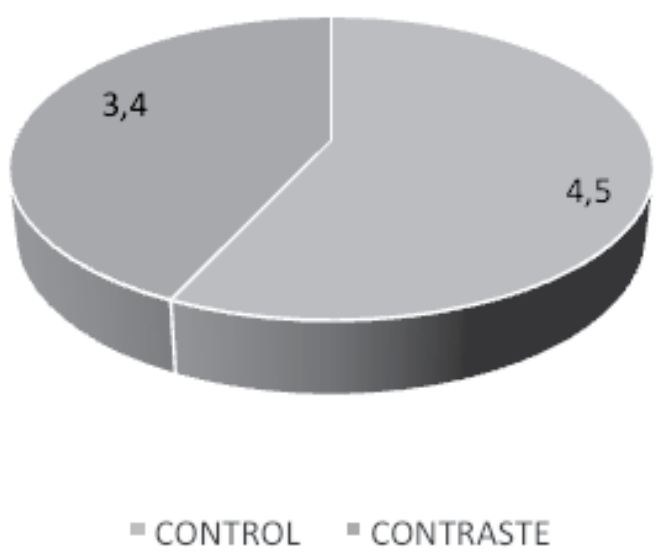

Por lo tanto, la interdisciplinariedad no es un tema común. Plantea la integración de saberes superando la disciplinariedad, la parcelación y fragmentación del conocimiento, producto de la división del trabajo. Corresponde a los educadores abordarlo con urgencia para construir estadios interdisciplinarios que trasciendan a metodologías avanzadas, donde los alumnos desarrollan mucho mejor sus estructuras conceptuales, permitiendo una mejor apropiación de los conocimientos, a través de las competencias psicomotrices que no sufren variaciones significativas por la aplicación de una clase interdisciplinar, pero el poder complementarla con el conocimiento de otras áreas le da un mayor sustento o soporte al aprendizaje. Por esta razón, las competencias socio-afectivas juegan un papel determinante en la relación con las demás competencias, siendo 
Jose Salvador Pagano B., Carlos Alberto Pérez G. - Interdisciplinariedad entre educación física y ciencias naturales

la esencia misma de un proceso de enseñanza aprendizaje interdisciplinar.

Precisamente el propósito ha de ser, como se esboza en este trabajo, que los contenidos curriculares estén en sintonía con una constante superación de la separación entre asignaturas teóricas y prácticas para el desarrollo de estructuras mentales, que permitan una mejor y mayor construcción del conocimiento.

En consecuencia y acorde con los resultados obtenidos, la interdisciplinariedad no es la sumatoria de saberes, sino la integración, la articulación de disciplinas para producir soluciones a los diferentes problemas de aprendizajes, acaba con las posiciones individualistas de las áreas, permite un currículo más articulado y mejor dispuesto para el conocimiento globalizado.

La hipótesis de trabajo planteada en la investigación se cumple, por lo tanto, la efectividad de la interdisciplinariedad entre la Educación Física y las Ciencias Naturales en el mejoramiento de los conceptos básicos de ambas disciplinas en los niñas de tercer grado de básica primaria mejoró en un $41 \%$, lo que equivale a un mejoramiento de notas en 1,1 puntos promedio. Por ello, el área de Educación Física constituye una herramienta fundamental para aplicar interdisciplinariedad con las ciencias naturales, por su naturaleza que facilita la motivación y el manejo de conceptos y contenidos.

\section{Referencias bibliográficas}

Carvajal Y. (2010). Interdisciplinariedad: desafío para la educación superior y la investigación. Revista Luna Azul, 156-169.

Conde, J., Arteaga, M., y Viciana, V. (1998). Interdisciplinariedad de las áreas en educación primaria. La educación física refuerzo del área de lengua castellana y literatura. Apunts. Educación Física y Deportes, 51, 46-54.

Do Santos, M. M., y Campoy, T. J. A. (2010). Formación docente reflexiva: perspectivas interdisciplinarias en educación física. Revista Electrónica de Investigación y Docencia (REID), (3), 133-144.

Fogarty, R. (1991). Ten ways to integrate curriculum. Educational leadership, 49(2), 61-65.

Frega, A. L. (2007). Interdisciplinariedad: Enfoques Didácticos para la Educacion General. Editorial Bonum.

León, V. E. (1997). La Física integrada a la Agronomía. Evento Internacional de Pedagogía, 97.

Meriño, F. y Cabezas, M. (2011). La interdisciplinariedad en la formación inicial del maestro primario. Cuadernillo de educación y desarrollo, 3(29).

Piaget, J. (1978). Estudio de psicología genética. Buenos Aires: EMECE. 